\title{
Forschungspartizipation von Kindern zwischen Minimalbeteiligung und Entscheidungsmacht: Forschungsethische Herausforderungen und Perspektiven der Forschungssubjekte auf Forschung in Kindertagesstätten
}

\author{
Beatrice Rupprecht $\mathbb{D} \cdot$ Katrin Lattner $\mathbb{D}$
}

Eingegangen: 4. November 2020 / Angenommen: 2. November 2021 / Online publiziert: 13. Januar 2022 (C) Der/die Autor(en) 2022

Zusammenfassung Der Beitrag geht der Frage nach, in wieweit Kinder im Rahmen von Forschungsprojekten in Kitas beteiligt werden. Zur Beantwortung der Frage werden die Daten von zwei Studien herangezogen, die im Projekt „Forschung trifft Kita“ erhoben wurden. Die Ergebnisse der Interviews mit Kindern und die Ergebnisse der deutschlandweiten Befragung von pädagogischen Fach- und Leitungskräften zeigen, dass die Mitwirkungsmöglichkeiten von Kindern derzeit noch stark eingeschränkt werden. Im Kontext der Bewertung der ethischen Strenge der in Kitas durchgeführten Forschungsprojekte wird für Partizipation als ergänzendes Ethikkriterium plädiert.

Schlüsselwörter Partizipation · Kindertagesstätte $\cdot$ Forschungsethik $\cdot$ Kinderrechte

\footnotetext{
Dr. Beatrice Rupprecht $(\bowtie)$

Institut für Pädagogik und Didaktik im Elementar- und Primarbereich, Professur Schulpädagogik des Primarbereichs, Universität Leipzig, Marschnerstraße 31, 04109 Leipzig, Deutschland

E-Mail: beatrice.rupprecht@uni-leipzig.de

Dr. Katrin Lattner

Institut für Pädagogik und Didaktik im Elementar- und Primarbereich, Professur Pädagogik der frühen Kindheit, Universität Leipzig, Marschnerstraße 31, 04109 Leipzig, Deutschland E-Mail: katrin.lattner@uni-leipzig.de
} 


\title{
Research participation of children between minimal participation and decision-making power: research ethical challenges and perspectives of research subjects on research in day-care centers
}

\begin{abstract}
This paper examines the extent to which children are involved in research projects in Kindergartens. In order to answer this question, the data from two substudies that were collected in the project "Research Meets Kindergarten" are used. The results of interviews with children and the results of a nationwide survey of educational staff and heads show that children's opportunities for participation are currently still severely limited. In the context of evaluating the research ethical rigour of research projects conducted in Kindergartens, participation is advocated as a complementary ethical criterion.
\end{abstract}

Keywords Participation · Kindergarten · Research ethics · Children's rights

Die sozialwissenschaftliche Kindheitsforschung definiert „Kindheit (...) als eigene Lebensphase (...) und als Konstrukt generationaler Verhältnisse“ (Heinzel 2012, S. 9), die durch aktuelle Lebensumstände sowie Alltags- und Beziehungserfahrungen von Kindern geprägt ist. Diese aus der Perspektive der Kinder zu erforschen, setzte eine Diskussion um methodische und methodologische Herausforderungen in Gang (ebd.), in der u.a. die Beteiligung der Kinder an Bedeutung gewinnt. Die Hinwendung zum Kind und der Stärkung seiner aktiven Mitbestimmung im Forschungsprozess ist im Übereinkommen über die Rechte des Kindes verankert (UNKinderrechtskonvention). Hieraus geht hervor, dass der Wille und die Meinung des Kindes in allen sie betreffenden Angelegenheiten zu beachten ist (Artikel 12, Artikel 13). Daraus folgt logisch konsequent die Beteiligung von Kindern im Sinne ihrer Mitsprache in den Belangen einer Forschungsteilnahme, um ihrer Entscheidungsfreiheit und der Selbstbestimmung über die Art und das Ausmaß ihrer Beteiligung Rechnung zu tragen (Horgan 2017, S. 246). Treten Kinder als Akteure innerhalb von Forschung in Erscheinung (z.B. als Forschungssubjekte), dann ist dieses Mitsprache- bzw. Partizipationsrecht der Kinder durch die Forschenden zu wahren. Bislang wird der Partizipationsaspekt in den in Deutschland geltenden Ethikrichtlinien jedoch ausgeblendet.

\section{Forschungsethische Handlungsprämissen}

Die ethische Betrachtung von Forschung findet ihren Ausgangspunkt im internationalen, insbesondere im anglo-amerikanischen Raum. Unter Forschungsethik wird dabei konkret die ,auf Forschungshandeln angewandte Ethik“ verstanden, welche die „Grundprinzipien moralisch angemessenen Handelns für die empirische For- 
schungspraxis“ (RatSWD 2017, S. 8) determiniert. ${ }^{1}$ Im Kontext der Datenerhebung und Datenanalyse soll den an Forschungsprojekten Beteiligten sowie der Gesellschaft kein Schaden entstehen (ebd.). Die disziplinübergreifenden Erläuterungen in den Richtlinien umfassen die Aspekte Freiwilligkeit, Informiertheit und den Datenschutz (Döring und Bortz 2016b, S. 123 f.). Insbesondere für die direkte Interaktion mit Kindern innerhalb der Datenerhebung wird von Forscher/-innen ein hohes Maß an Reflexivität gefordert, um ein Gespür für potenziell gefährdende Situationen zu entwickeln und in diesen Situationen ethisch angemessen handeln zu können (Canosa et al. 2018, S. 411; Phelan und Kinsella 2013, S. 84).

Aktuell finden in Deutschland verschiedene Ethikrichtlinien Anwendung, die für die einzelnen Fachdisziplinen u. a. von den Dachverbänden erarbeitet wurden. Für die Forschung im Elementar- und Primarbereich dient der von der Deutschen Gesellschaft für Erziehungswissenschaft (DGfE) erarbeitete Ethik-Kodex als Orientierung. Zentral sind dabei das Gebot der Freiwilligkeit der Teilnahme und die damit verbundene informierte Einwilligung durch die Teilnehmenden sowie die vertrauliche Behandlung der erhobenen Daten zum Schutz der Integrität der Teilnehmenden (DGfE 2016, S. 3). In diesem Zusammenhang betonen Christensen und Prout (2002), dass die reine Einhaltung der Ethikrichtlinien für die Forschung mit Kindern nicht hinreichend ist - vielmehr muss eine ethische Symmetrie angestrebt werden, welche strategisch die Interessen, Gefühle und insbesondere die Rechte der Kinder als denen von Erwachsenen gleichwertig gegenüberstellt (S. 492f.). Das entspricht im Kern auch Artikel 12 der UN-Kinderrechtskonvention („Partizipations-Artikel“, Horgan 2017, S. 247), worin die Notwendigkeit begründet werden kann, die forschungsethischen Kriterien um den Aspekt der Partizipation zu erweitern (Canosa et al. 2018, S. 400).

\section{Partizipation und partizipative Forschung}

Das allgemeine, rechtlich verankerte Partizipationsrecht der Kinder ist von der sog. partizipativen Forschung abzugrenzen, die im Zuge der methodologischen Weiterentwicklung empirischer Sozialforschung als eigener Forschungsstil hervorging. Bei Letzterem geht es darum, ,die soziale Wirklichkeit partnerschaftlich [zu] erforschen“ (von Unger 2014, S. 1). Der partizipative Einbezug von Kindern zeigt positive Effekte für ihre Gesundheit (Naidoo und Wills 2003), das Demokratiebewusstsein (Bruner et al. 2001) und das Selbstbewusstsein (Ballaschk und Anders 2020). Doch ist nicht jede Forschung, die Kindern eine Beteiligung jenseits der Informationslieferung im Datenerhebungsprozess einräumt, automatisch auch partizipative Forschung.

Das Stufenmodell der Partizipation nach Wright et al. (2010) hebt die Entscheidungsmacht der Akteure hervor, die ihnen in allen Phasen eines Forschungsprojektes in unterschiedlichem Maße von Seiten der Forscher/-innen zugestanden wird. Die neun aufeinander aufbauenden Stufen des Modells verdeutlichen dabei verschiede-

\footnotetext{
${ }^{1}$ Döring und Bortz (2016a) weisen die Wissenschafts- und Forschungsethik als einen von vier Standards der Wissenschaftlichkeit aus, wobei die ethische Strenge als Kriterium der wissenschaftlichen Qualität beschrieben wird (S. 90f.).
} 
ne Ausprägungsformen: Nicht-Partizipation, Vorstufen der Partizipation, Partizipation, Geht über Partizipation hinaus. Die Stufe der Nicht-Partizipation wirkt dem Grundprinzip der Beteiligung entgegen. Erst mit Erreichung der Vorstufen wird ein Forschungsprojekt für die Beteiligung der Akteure geöffnet (durch „Information, Anhörung und Einbeziehung“, von Unger 2014, S. 40). Vollendung findet Partizipation im Beginn der partizipativen Forschung, also in der zunehmenden aktiven Involvierung der Akteure in die zentralen Entscheidungsprozesse eines Projekts (ebd.). In der Forschungspraxis werden Akteure jedoch vorwiegend auf den Vorstufen der Partizipation beteiligt, d.h. sie werden informiert, befragt und z. B. in beratender Funktion einbezogen (Wilkinson und Wilkinson 2017, S. 219). Jacquez et al. (2013) zeigten, dass von 399 betrachteten Studien mit Kindern, die den Ansatz der kommunikationsbasierten partizipativen Forschung nutzten, lediglich $10 \%$ auf einer partnerschaftlichen, mitbestimmenden Partizipationsstufe einordbar waren (S. 178). Dabei bezog sich die Beteiligung vorrangig auf die Identifizierung der Bedarfe, Prioritäten und Zielstellungen der Projekte, des Forschungsdesigns und der Durchführung (ebd., S. 179).

Partizipative Forschung findet jedoch erst statt, wenn die Praxispartner/-innen ,die Projektgestaltung mitbestimmen können (Stufe 6), die einzelnen Projektkomponenten in eigener Regie umsetzen (Stufe 7) und/oder wichtige Projektentscheidungen im Wesentlichen selbst vornehmen (Stufe 8)“ (von Unger 2014, S. 40). Bezugnehmend auf das Modell können Kinder in Kitas also entweder minimal (auf Stufe 3 bis 5) oder als Co-Forscher/-innen maximal (auf Stufe 6 bis 8) an der Gestaltung und Durchführung der Forschung mitwirken (von Unger 2014).

Zwischen der Minimalbeteiligung einerseits und der Entscheidungsmacht andererseits lässt sich ein Spannungsfeld verorten. Die mit der Partizipation von Kindern einhergehende Demokratisierung des Forschungsprozesses (Canosa et al. 2018, S. 402) bedarf dabei einer Offenheit und Flexibilität der Forschenden (AGJ 2020) sowie deren Bereitschaft, Kindern Freiräume für ihre Entscheidungen zu schaffen und die sie betreffenden Angelegenheiten verständlich zu artikulieren (Thomas und O’Kane 1998). Den Möglichkeiten der aktiven Mitgestaltung von Lebens- und Lernerfahrungen, der Selbstbefähigung und Meinungsäußerung auf Seiten der Kinder (Moser 2012) stehen u. a. ein großer zeitlicher Aufwand, aber auch Unsicherheiten im Umgang mit dem partizipativen Vorgehen seitens der Forscher/-innen gegenüber (von Unger 2014, S. 85 f.).

\section{Zusammenhänge der Ethik und Partizipation im Forschungskontext}

Nentwig-Gesemann und Großmaß diskutieren auf der Basis des DGfE-Ethik-Kodexes (2016) und der UN-Kinderrechtskonvention folgende Handlungsprämissen, um die Mitbestimmung und Persönlichkeitsrechte von Kindern in Forschungsprojekten zu sichern (Nentwig-Gesemann und Großmaß 2017, S. 218):

1. Verwendung eines Methodenrepertoires, das Kindern vielseitige Möglichkeiten der verbalen und non-verbalen Teilnahme einräumt, 


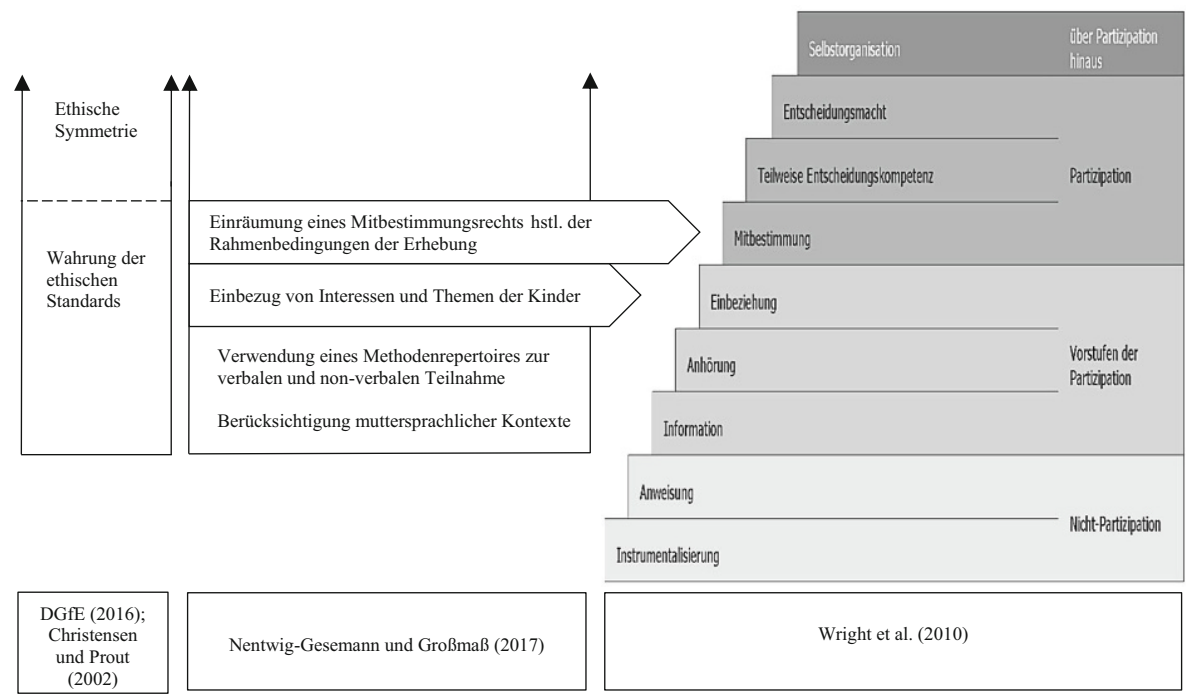

Abb. 1 Modellierung ethischer und partizipativer Anforderungen im Forschungskontext (in Anlehnung an Wright et al. 2010, S. 42; Nentwig-Gesemann und Großmaß 2017, S. 218; DGfE 2016; Christensen und Prout 2002)

2. Berücksichtigung etwaiger muttersprachlicher Kontexte der Kinder (Datenerhebung) sowie

3. Einbezug von Interessen und Themen der Kinder durch geeignete Methoden (bspw. Interviews, Zeichnungen),

4. Einräumung eines Mitbestimmungsrechts hinsichtlich der Rahmenbedingungen der Erhebung (Ort, Zeit etc.).

Diese forschungsethisch relevanten Partizipationsaspekte lassen sich ebenso wie die anderen Ethikkriterien unterschiedlichen Stufen des Partizipationsmodells (Wright et al. 2010) zuordnen, wie Abb. 1 veranschaulicht.

Die Einhaltung der Ethik-Richtlinien kann Modellstufe 3 (Information) zugeordnet werden: Forschungsprojekte mit Kindern wahren Artikel 12 und Artikel 13 der UN-Kinderrechtskonvention also dann, wenn Kinder freiwillig teilnehmen und alle relevanten, sie betreffenden Informationen hinsichtlich der Teilnahme erhalten (gemäß dem ethischen Kriterium der informierten Einwilligung). Würden Kinder aufgrund einer $\mathrm{Zu}$ - oder Anweisung an einem Projekt teilnehmen, ohne selbst die Möglichkeit zur Einwilligung oder Ablehnung zu haben, ist von einer Nicht-Partizipation auszugehen und die Wahrung der Kinderrechte anzuzweifeln (NentwigGesemann und Großmaß 2017, S. 219). Daraus folgt, dass mit der Erreichung dieser Stufe des Partizipationsmodells ein Minimalgrad der Partizipation und zugleich die Absicherung der Mindestanforderungen einer ethischen Strenge erzielt werden. Die ersten zwei Handlungsprämissen nach Nentwig-Gesemann und Großmaß (2017) entsprechen den Stufen 3 und 4 des Partizipationsmodells (Information, Anhörung). Sie bilden die Grundlage für die Mitteilung, Verständigung und Beteiligung der Kinder und sind Voraussetzung für die von Christensen und Prout (2002, S. 492f.) 
geforderte ethische Symmetrie zwischen Erwachsenen und Kindern innerhalb des Forschungsprozesses. Der Einbezug der konkreten Themen und Interessen der Kinder im Projekt und ihre Berücksichtigung im methodischen Design (dritte Handlungsprämisse) steigert den Grad der Partizipation sowie den der ethischen Strenge, da die Kinder mittels ihrer Interessen und Themen stärker repräsentiert und damit integriert werden (Stufe 5 des Partizipationsmodells). Demgegenüber ist die vierte Handlungsprämisse (Mitbestimmung) auf Modellstufe 6 einzuordnen. Sie beschreibt eine Realisierungsform der partizipativen Forschung, ab der die ethische Symmetrie vollendet wird.

Zwar wurde im internationalen Kontext die Beteiligung von Kindern bzw. die partizipative Forschung mit Kindern hinsichtlich der methodischen Herausforderungen beleuchtet, bspw. in Bezug auf das Forschungsdesign (u. a. Jacquez et al. 2013), die Machtstrukturen zwischen Erwachsenen und Kindern (z. B. Horgan 2017), die Minimierung sozialer Distanz (z. B. Raffety 2015) oder die Steigerung der Datenqualität (z.B. Cheney 2011). Bisher fehlen jedoch Studien, die die Wahrung der Kinderrechte zum Gegenstand der Forschung machen. Die Frage danach, inwieweit die Kinderrechte in Forschungsprojekten mit Kindern grundsätzlich gewahrt werden, ist als unbeantwortet zu deklarieren. Dieses Desiderat wird im Forschungsprojekt „Forschung trifft Kita“ aufgegriffen.

\section{Das Projekt ,Forschung trifft Kita“}

Das Forschungsprojekt befasst sich damit, wie Kita-Akteure die Teilnahme an Forschung erleben und inwieweit aus ihrer Sicht die ethische Strenge in Forschungsprojekten in der Kita erreicht wird. Im Beitrag wird eine Teilfrage beleuchtet: Wie werden die Rechte der Kinder gemäß der UN-Kinderrechtskonvention (Artikel 12 und Artikel 13) in Forschungsprojekten mit Kindern in Kitas durch Forscher/-innen gewahrt? Wie oben diskutiert wurde, bezieht das gleichermaßen die Einhaltung forschungsethischer Kriterien (Freiwilligkeit der Teilnahme, informierte Einwilligung, Datenschutz resp. Integrität der Teilnehmenden) sowie die Partizipation ein.

Das explorativ ausgerichtete Projekt ist als Mixed Methods Design (,,sequentielles qualitativ-quantitatives Design“, Kelle 2014, S. 161) konzipiert. Die Forschungsfrage wird dabei aus der Sicht der Kinder als Teilnehmende und aus der Sicht von Fachund Leitungskräften als Gatekeeper beantwortet. Dazu werden die Daten aus zwei Teilstudien herangezogen.

\section{Erhebungsmethoden}

Zur Beantwortung der Fragestellung werden die Aussagen von Kindern aus den Interviews (Kinderstudie, Frühjahr 2017) und ausgewählte Items der Online-Befragung von Fach- und Leitungskräften (Fach- und Leitungskräftestudie, Sommer 2019) ausgewertet.

Die Kinderstudie umfasst halbstrukturierte, leitfadengestützte Gruppeninterviews (Trautmann 2010). Die teilnehmenden Kinder konnten ihre Eindrücke und das Er- 
leben des Beforscht-Werdens unmittelbar im Anschluss an die eigenen Erfahrungen in der Teilnahme an Forschung kommunizieren, um dem Verblassen oder Verändern der Erinnerungen in retrospektiven Erzählungen im Zeitverlauf entgegenzuwirken. Die Interviews schlossen dazu unmittelbar an eine Untersuchung von Studierenden zur Erfassung der kindlichen Vorstellungen von Schule im Rahmen des Grundschullehramtsstudiums an der Universität Leipzig an. In den Gruppeninterviews standen vorwiegend die Einzelmeinungen der Kinder im Fokus, ohne Beachtung gruppendynamischer Prozesse und kollektiver Phänomene (Vogl 2015). Gleichzeitig wirkten die Gespräche durch die untergeordnete Anzahl der Forscher/-innen einer generationalen Dominanz entgegen und boten durch die Einbeziehung mehrerer Kinder derselben Vorschulgruppe eine vertraute, auf Offenheit und Kommunikation ausgerichtete Erhebungssituation (Vogl 2015, S. 64). Die Interviews dauerten bis zu 14 min und fanden in einem ungestörten Raum der Kitas statt.

Die Sichtweise der Gatekeeper wurde in der Fach- und Leitungskräftestudie mittels einer deutschlandweiten Online-Befragung von pädagogischen Fach- und Leitungskräften in Kitas erhoben. Vom Leibniz Institut für Sozialwissenschaften (GESIS) wurde dazu eine repräsentative, dreigeschichtete Zufallsstichprobe aus Kitas in 100 Gemeinden aus allen 16 Bundesländern gezogen. Auf der Grundlage von 11.409 Kitas wurden per Zufallsalgorithmus 9135 Kitas ausgewählt und kontaktiert. Alle Kitas erhielten ein Anschreiben mit allen wichtigen Informationen zum Forschungsprojekt.

\section{Erhebungsinstrumente}

Der entwickelte Interviewleitfaden der Kinderstudie umfasst Fragen zu folgenden Themenaspekten: Erfahrungen mit dem Forschungssetting, Vorstellungen der Kinder von „Forscherinnen und Forschern“", Aufgaben der Kinder während der Erhebung, Ankündigung der Erhebung sowie Kommunikation mit Eltern über Forschungserfahrungen. Allen Interviews lag derselbe halbstrukturierte Leitfaden zugrunde.

In der Online-Befragung wurde ein Fragebogen eingesetzt, der iterativ und induktiv-deduktiv auf der Grundlage der Ergebnisse der Kinderstudie, der Ergebnisse der zuvor geführten leitfadengestützten Interviews mit pädagogischen Fachkräften $(N=5)$ und des aktuellen Forschungsstands entwickelt wurde. Die Items zur Erfassung der ethischen Strenge und der Partizipation der Kinder sind stringent von den Ethikrichtlinien der DGfE (2016, S. 3), den Erläuterungen der zentralen Ethik-Kriterien in Döring und Bortz (2016b, S. 124f.) sowie den oben angezeigten ethischen Handlungsprämissen nach Nentwig-Gesemann und Großmaß (2017, S. 218) abgeleitet. Die Items lassen sich sieben Schwerpunkten zuordnen: Allgemeine Angaben, Grundhaltung zur Forschungsteilnahme, Nutzen der Teilnahme, Einschätzungen zur ethischen Strenge, wahrgenommene Störfaktoren, zukünftige Forschung in der Kita sowie Informationsverhalten über Forschungsergebnisse. Die Pilotierung des Entwurfs erfolgte mittels eines Standardbeobachtungs-Pretests (Porst 2014) $(N=30)$ und eines zweistufigen kognitiven Pretests mit pädagogischen Fach- und Leitungskräften $(N=4)$, woraus eine inhaltliche Anpassung des Fragebogens resultierte. Dieser wurde anschließend in das Portal SoSci Survey (Leiner 2019) eingepflegt und eine PHP- 
Filterführung zur Steuerung der Befragung in Abhängigkeit der beruflichen Position (Kita-Leiter/-innen vs. pädagogische Fachkräfte) und der Forschungserfahrungen eingerichtet.

\section{Stichproben}

Die Kinderstudie umfasst sechs Gruppen von Fünf- bis Sechsjährigen ( $N=17$, davon $n=9$ Jungen und $n=8$ Mädchen) aus drei Leipziger Kitas. Fünf Gruppen bestanden aus drei Kindern, eine Gruppe aus zwei Kindern (ein Kind war am Erhebungstag erkrankt). Die Auswahl der Kinder erfolgte im Rahmen der Erhebung der Studierenden anhand festgelegter Kriterien, wozu das Alter der Kinder (5- bis 6-Jährige) und die Zugehörigkeit zur Vorschulgruppe zählen.

Die Stichprobe der Online-Befragung umfasst insgesamt $N=1200$ Personen, von denen $n=953$ Personen $(87 \%)$ als Leitungskraft (mit vollständiger oder anteili-

Tab. 1 Überblick der Kategorien und Subkategorien für die induktiv-deduktiv gewonnenen Themenaspekte der Forschungserfahrungen von Kindern in Kitas

\begin{tabular}{|c|c|}
\hline Kategorien & Subkategorien \\
\hline \multirow[t]{2}{*}{ 1. Wiederholung der Erhebung } & 1.1. Keine Wiederholung \\
\hline & 1.2. Wiederholung \\
\hline \multirow[t]{6}{*}{ 2. Ankündigung der Erhebung } & 2.1 Ankündigung durch die Eltern \\
\hline & 2.2 Keine Ankündigung durch die Eltern \\
\hline & 2.3 Ankündigung durch die Fachkräfte \\
\hline & 2.4 Keine Ankündigung durch die Fachkräfte \\
\hline & 2.5 Vom Kind selbst gewusst \\
\hline & $\begin{array}{l}2.6 \text { Grund für fehlende Ankündigung durch die } \\
\text { Fachkräfte }\end{array}$ \\
\hline \multirow{2}{*}{$\begin{array}{l}\text { 3. Kommunikation mit den Eltern über die } \\
\text { Erhebung }\end{array}$} & 3.1 Absicht \\
\hline & 3.2 Erzählinhalte \\
\hline \multirow[t]{2}{*}{ 4. Befinden der Kinder während der Erhebung } & 4.1 Wohlbefinden \\
\hline & 4.2 Unwohlsein \\
\hline \multirow[t]{3}{*}{$\begin{array}{l}\text { 5. Teilnahmeeinverständnis der Kinder zur } \\
\text { Erhebung }\end{array}$} & $\begin{array}{l}\text { 5.1 Frage nach dem Einverständnis durch Fach- } \\
\text { kräfte }\end{array}$ \\
\hline & $\begin{array}{l}\text { 5.2 Keine Frage nach dem Einverständnis durch } \\
\text { die Eltern }\end{array}$ \\
\hline & $\begin{array}{l}\text { 5.3 Frage nach dem Einverständnis - kein Wissen } \\
\text { „wer“" }\end{array}$ \\
\hline \multirow[t]{2}{*}{ 6. Begriffsverständnis Forscher/-in } & 6.1 Tätigkeit von Forschern/-innen \\
\hline & 6.2 Untersuchungsgegenstand \\
\hline $\begin{array}{l}\text { 7. Erleben der Anwesenheit fremder Personen in } \\
\text { der Kita }\end{array}$ & - \\
\hline 8. Kontakt mit technischen Geräten in der Kita & - \\
\hline 9. Dauer der Erhebung & - \\
\hline 10. Aufgaben der Kinder während der Erhebung & - \\
\hline 11. Anwesenheit von Forschern/-innen in der Kita & - \\
\hline 12. Incentives & - \\
\hline
\end{tabular}


ger Freistellung) und $n=147$ Personen (13\%) als pädagogischen Fachkraft (ohne Leitungsfunktion) tätig waren. Das Durchschnittsalter beträgt zum Zeitpunkt der Erhebung 46,9 Jahre $(S D=10,54)$. Aufgrund der adaptiven Filterführung der Online-Befragung wurden nicht alle Fragen auch allen Teilnehmenden gestellt. Daraus ergeben sich Abweichungen der fragebezogenen Stichprobengrößen, auf die in der Ergebnisdarstellung hingewiesen wird.

\section{Auswertungsmethoden}

Die Namen der Kinder aus den Interviews wurden vor der Auswertung anonymisiert. Alle Interviews wurden transkribiert und mittels der inhaltlich strukturierenden qualitativen Inhaltsanalyse im Sieben-Schritt-Verfahren nach Kuckartz (2018, S. $100 \mathrm{ff}$.) im Analyseprogramm MAXQDA (Version 11) analysiert. Ausgehend vom Material wurde ein Kodierungssystem mit 12 induktiv-deduktiv gewonnenen thematischen Kategorien entwickelt, die in Tab. 1 aufgeführt sind.

Die Auswertung der in der Online-Befragung anonym erhobenen Daten erfolgte mit SPSS (Version 25). Die Häufigkeitsverteilung der beruflichen Position der Befragten ergab eine Ungleichgewichtung der Anteile von Leitungskräften und pädagogischen Fachkräften. Um einer Verzerrung der Ergebnisse entgegenzuwirken, wurden die Daten gewichtet, wobei der Gewichtungsfaktor aus der Population der entsprechenden Berufsgruppen anhand der Statistiken der Kinder- und Jugendhilfe (Statistisches Bundesamt 2020) ermittelt wurde. Er beträgt für die Gruppe der Leitungskräfte 0,07 und für die Gruppe der pädagogischen Fachkräfte (ohne Leitungsfunktion) 7,71 .

\section{Ergebnisse}

\subsection{Wie erleben Kinder die Teilnahme an Forschungsprojekten?}

Die Interviews liefern einen Einblick in die Erfahrungswelt der Kinder zum subjektiven Erleben des Beforschtwerdens. Dabei variieren die Anzahl und Ausführlichkeit der Aussagen der Kinder innerhalb der 12 Themenkategorien. Einzelne Aspekte des Erlebens von Forschung treten wiederholt auf.

In der Kategorie Teilnahmeeinverständnis der Kinder zur Erhebung zeigt sich, dass dieses ethische Kriterium nicht von allen Kindern als gewahrt erlebt wird. Diese enthält Textpassagen, in der z. B. die Mutter eines Kindes die Entscheidung zur Teilnahme an der Untersuchung traf, ohne dessen Einwilligung vorab zu erfragen, was einer Anweisung gleicht (Nicht-Partizipation, Wright et al. 2010). Gruppe B_002, Zeile 117-118: „Interviewerin 1 (I1): Die Mama hat dich gefragt, Konrad? Konrad: Die Mama hat mich nicht gefragt. Die hat mich einfach angemeldet. “

Andere Kinder berichteten demgegenüber vereinzelt, von pädagogischen Fachkräften in den Entscheidungsprozess einbezogen worden zu sein, indem diese im Vorfeld der Erhebung das Einverständnis der Kinder abfragten (Einbeziehung, Vorstufen der Partizipation, Wright et al. 2010). Hierzu äußerte ein Kind, gefragt worden 
zu sein, ohne die konkrete Person zu erinnern. Dass eher die Erfahrung des GefragtWerdens als die sie befragende Person in Erinnerung blieb, könnte auf die aktive Rolle des Kindes im Entscheidungsprozess und der damit einhergehenden Selbstwirksamkeit zurückgeführt werden.

Die Unterschiedlichkeit an Erfahrungen spiegelt sich auch in der Kategorie $A n$ kündigung der Erhebung wider, die im Sinne des Informierens als Vorstufe der Partizipation gilt (Wright et al. 2010): Einige Kinder erinnerten sich, von den Fachkräften oder Eltern über den Besuch der Forscher/-innen informiert worden zu sein. Dies erfuhren sie teils erst in dem Moment des Eintretens der Forscher/-innen in die Kita, teils im Vorfeld. Im Prozess des Überlegens, durch wen und wann die Kinder von der Erhebung erfuhren, äußerte im Interview ein Kind, von sich aus gewusst zu haben, dass Forscher/-innen zu ihm kommen würden. Es scheint dem Kind wichtig zu sein, selbst der wissende Akteur und damit unabhängig von Eltern bzw. Fachkräften zu sein, um mit den Forscher/-innen in Beziehung zu treten. Gruppe B_002, Zeile 25-27: „I 1: (...) Und hat eure Mama oder euer Papa euch erzählt, dass wir heute zu euch kommen? (...) Konrad: Ich wusste es schon selber. “

Andere Kinder berichteten, vorab weder von den pädagogischen Fachkräften noch von den Eltern informiert worden zu sein. Weshalb die Fachkräfte bzw. Eltern den Kindern die Information zum Besuch vorenthielten, erklären sich zwei Kinder damit, dass sie es entweder vergessen haben oder für die Kinder eine Überraschung planten (Unterkategorie Gründe für fehlende Ankündigung). Gruppe 001, Zeile 29-32: „I 1: Hat eure Erzieherin euch erzählt, dass wir heute kommen? Alle Kinder antworten: Nö. (...) Julian: Ich glaube, ich weiß warum sie uns das nicht gesagt hat. Damit das eine Überraschung ist. Das glaube ich. “

Dabei stellte sich heraus, dass ein Kind bereits vor seiner Teilnahme an der Kinderstudie anderweitig Forschungserfahrungen in der Kita gesammelt hatte. In seinem kurzen Erfahrungsbericht verzichtet er auf die konkrete Zuschreibung des Begriffes „Forscher/-in“ und spricht durchweg von der ihn besuchenden „Frau“. Während ihm sowohl die (Forschungs-)Tätigkeiten als auch die erfolgreiche Bewältigung der ihm übertragenen Aufgabe (Rätselkönig werden) samt Belohnung in Erinnerung blieb, gerieten der Name bzw. die äußerliche Erscheinung der Forscherin in Vergessenheit. Somit sind diejenigen Charakteristika einer Erhebungssituation für eine Forschungserfahrung von Bedeutung, in denen das Kind selbst aktiv (eingebunden) war, etwas praktisch tun konnte (Rätseln) und mitentscheiden durfte (Auswahl der Belohnung). Folglich ist anzunehmen, dass Situationen aus Sicht des Kindes weniger erwähnenswert scheinen, in denen es passiv blieb, z. B. während sich die „Frau“ vorstellte oder den Ablauf erklärte.

\subsection{Wie wurden die Rechte der Kinder aus Sicht der pädagogischen Fach- und Leitungskräfte in bisherigen Forschungsprojekten durch Forscher/ -innen gewahrt?}

Zur Beantwortung der Frage werden die Ergebnisse der Fach- und Leitungskräftestudie (Online-Befragung) dargestellt und die Antworten zu der Skala „Forschungsethik Kinder" sowie zur Skala „Partizipation Kinder“ herangezogen. Die Skalen wurden auf der Grundlage einzelner Items zur Messung der unterschiedlichen Ausprägungs- 
Tab. 2 Häufigkeitsverteilungen der Einschätzung von befragten pädagogischen Fach- und Leitungskräften zur der Forschungsethik ${ }^{\mathrm{a}}$ durch die ForscherInnen bezugnehmend auf das letzte Forschungsprojekt, bei dem Kinder in der Einrichtung teilnahmen (absolute Häufigkeiten, prozentuale Verteilungen in Klammern)

\begin{tabular}{lllll}
\hline Forschungsethikaspekt & $\begin{array}{l}\text { Gültige } \\
\text { Antworten }\end{array}$ & $\begin{array}{l}\text { Einschätzung } \\
\text { Ja }\end{array}$ & Nein & Summe \\
\hline Information über Ziele der Erhebung & 156 & 65 & 91 & 156 \\
& & $(41,7 \%)$ & $(58,3 \%)$ & $(100,0 \%)$ \\
Information über Methode der Erhebung & 164 & 97 & 67 & 164 \\
& & $(59,1 \%)$ & $(40,9 \%)$ & $(100,0 \%)$ \\
Zusicherung der Vertraulichkeit der Aus- & 143 & 65 & 78 & 143 \\
sagen, Ergebnisse etc & & $(45,5 \%)$ & $(54,5 \%)$ & $(100,0 \%)$ \\
Zusicherung der Anonymisierung der & 139 & 56 & 83 & 139 \\
erhobenen Daten & & $(40,3 \%)$ & $(59,7 \%)$ & $(100,0 \%)$ \\
Information über Möglichkeit des Teil- & 156 & 98 & 58 & 156 \\
nahmeabbruchs & & $(62,8 \%)$ & $(37,2 \%)$ & $(100,0 \%)$ \\
Information zur Nutzung der erhobenen & 146 & 81 & 65 & 146 \\
Daten & & $(55,5 \%)$ & $(44,5 \%)$ & $(100,0 \%)$ \\
Information über Möglichkeit der Daten- & 128 & 30 & 98 & 128 \\
löschung & & $(23,4 \%)$ & $(76,6 \%)$ & $(100,0 \%)$ \\
Information über Ansprechpartner bei & 146 & 85 & 61 & 146 \\
Fragen zum Projekt & & $(58,2 \%)$ & $(41,8 \%)$ & $(100,0 \%)$ \\
$\Sigma$ & 1178 & 577 & 601 & 630 \\
& $(100,0 \%)$ & $(49,0 \%)$ & $(51,0 \%)$ & $(100,0 \%)$ \\
\hline
\end{tabular}

${ }^{a}$ Im Fragebogen wurde den Teilnehmenden dazu folgende Frage gestellt: Bitte denken Sie an das letzte Forschungsprojekt, an dem Kinder Ihrer Gruppe beteiligt waren. Wurden die Kinder von den Forscher/innen über folgende Punkte informiert?

grade der Forschungsethik resp. der Partizipation gebildet (Pospeschill 2013, S. 114). Die Items der Skalen wurden nur Teilnehmenden gestellt, die Erfahrungen in der Funktion als Gatekeeper in der Kita haben $(n=277,23 \%$ der Befragten).

Die Skala „Forschungsethik Kinder“ ( $\alpha=0,889$, basierend auf $n=96$ Fällen) besteht aus acht Items: Information über Ziele der Erhebung, Information über Methode der Erhebung, Zusicherung der Vertraulichkeit der Aussagen, Ergebnisse etc., Zusicherung der Anonymisierung der erhobenen Daten, Information über Möglichkeit des Teilnahmeabbruchs, Information zur Nutzung der erhobenen Daten, Information über Möglichkeit der Datenlöschung sowie Information über Ansprechpartner bei Fragen zum Projekt. Bezugnehmend auf das Partizipationsmodell (Wright et al. 2010) beziehen sich alle Items auf die dritte Modellstufe (Informieren).

Die Ergebnisse der Skala „Forschungsethik Kinder“ (siehe Tab. 2) zeigen, dass von den Befragten die Wahrung der forschungsethischen Standards unterschiedlich eingeschätzt wird. Für fünf der acht Skalenitems liegt die Verteilung mehrheitlich im Bereich der Wahrung der ethischen Strenge, d.h. die Befragten schätzten die Information über die Methode der Erhebung zu 59,1\% $(n=97)$, die Information über die Möglichkeit des Teilnahmeabbruchs zu 62,8\% $(n=98)$, die Information zur Nutzung der erhobenen Daten durch die Forscher/-innen zu 55,5\% $(n=81)$ und die Information über die Ansprechpartner/-innen im Projekt bei Fragen zu 58,2\% $(n=85)$ als zutreffend ein. Für vier Skalenitems gaben die befragten Personen mehrheitlich an, dass aus ihrer Einschätzung heraus die Kinder von den Forscher/-innen nicht über 
die Ziele der Erhebung ( $n=91,58,3 \%)$ oder die Möglichkeiten der Löschung ihrer Daten $(n=98,76,6 \%)$ informiert und ebenso weder die vertrauliche Behandlung der Daten $(n=78,54,5 \%)$ noch deren Anonymisierung $(n=83,59,7 \%)$ zugesichert wurde.

Zur Einschätzung des Grades der ethischen Strenge kann auf der Grundlage der Skala „Forschungsethik Kinder“ ein Index als arithmetisches Mittel der Einschätzung über alle acht Items errechnet werden. Aufgrund der Anzahl der Skalenitems kann der $\mathrm{I}_{\mathrm{FESK}}$ einen Wert zwischen 0,0 bis 8,0 annehmen. Dieser Index wird an dieser Stelle vorgeschlagen, da bislang eine empirische Festlegung fehlt, was konkret mit einem hohen Grad bzw. einem ,,besonders hohen Grad an ethischer Strenge“ (Döring 2015, S. 17) als Zielgröße gemeint ist. Auf der Grundlage des skalenbezogenen Indexes wird die Einteilung von vier Stufen empfohlen: Nicht vorhanden $\left(\mathrm{I}_{\mathrm{FESKi}}=0,0\right)$, niedrig $\left(0,0<\mathrm{I}_{\mathrm{FESKi}} \geq 3,0\right)$, mittel $\left(3,0<\mathrm{I}_{\mathrm{FESKi}} \geq 5,5\right)$ und hoch $\left(5,5<\mathrm{I}_{\mathrm{FESKi}} \geq 8,0\right)$.

Ergänzend zur Häufigkeitsverteilung der Skalenitems in Tab. 2 wurde für alle Fälle, welche alle acht Items bewertet haben, dieser Indexwert $\mathrm{I}_{\mathrm{FESK}}$ über alle eingeschätzten Forschungsprojekte berechnet $\left(N=188, M=4,67, S D=1,83, \sigma^{2}=3,33\right)$. In Bezug auf die oben beschriebene Einteilung der Grade zeigt sich im Durchschnitt ein mittlerer Grad der ethischen Strenge. Der berechnete Eta-Koeffizient $(0,079)$ legt dabei keine Abhängigkeit zwischen der beruflichen Position (Leiter/-innen vs. pädagogische Fachkräfte ohne Leitungsfunktion) und der Höhe des Indexwertes nahe $(F=1,55, p=0,21, T=1,08, d f=186)$.

Die zweite Skala, „Partizipation Kinder“ ( $\alpha=0,618$, basierend auf $N=89$ Fällen), besteht aus insgesamt sechs Items: Erfassung der Einwilligung in die Teilnahme, Möglichkeit der Mitbestimmung der Rahmenbedingungen, Beachtung der Interessen und Themen der Kinder, Abstimmung der Erhebungsmethode auf sprachliche und nicht-sprachliche Ausdrucksmöglichkeiten sowie Beachtung der Muttersprache (falls erforderlich). Die Skalenitems lassen sich unterschiedlichen Stufen des Partizipationsmodells (Wright et al. 2010) zuordnen. Während das Item Mitbestimmung der Rahmenbedingungen der Erhebung auf der sechsten Stufe eingeordnet und damit als Teil partizipativer Forschung verstanden werden kann, beziehen sich die restlichen Items auf die vierte Stufe (Vorstufen der Partizipation).

Die Ergebnisse der Skala in Tab. 3 zeigen, dass von den Befragten die Realisierung von Partizipation in Abhängigkeit des Partizipationsaspekts unterschiedlich eingeschätzt wurde. Für alle Skalenitems, die der Vorstufe von Partizipation zuzuordnen sind, schätzten die pädagogischen Fach- und Leitungskräfte mehrheitlich ein, dass im Rahmen der Forschungsprojekte die Kinder explizit zu ihrer Einwilligung befragt $(n=141,80,1 \%)$, die Interessen und Themen der Kinder berücksichtigt $(n=89,57,4 \%)$, die Erhebungsinstrumente auf die sprachlichen und nicht-sprachlichen Ausdrucksmöglichkeiten der Kinder angepasst wurden $(n=160,91,4 \%)$ und ihre Muttersprache bei der Erhebung Berücksichtigung fand $(n=85,61,6 \%)$. Allerdings wurde die Mitbestimmung der Rahmenbedingungen durch die Kinder im Forschungsprojekt mehrheitlich als nicht zutreffend eingeschätzt $(n=118,72,8 \%)$.

Auf der Grundlage aller Fälle, für die Antworten zu allen sechs Items vorlagen, wurde der Index für die Partizipation von Kindern $\left(\mathrm{I}_{\mathrm{PartK}}\right)$ als arithmetisches Mittel aus der Bewertung der sechs Skalenitems über alle Fälle ermittelt $(N=204, M=3,25$, 
Tab. 3 Häufigkeitsverteilungen der Einschätzung von befragten pädagogischen Fach- und Leitungskräften zu den gewährten Partizipationsmöglichkeiten von Kindern durch die ForscherInnen ${ }^{\text {a }}$ bezugnehmend auf das letzte Forschungsprojekt, bei dem Kinder in der Einrichtung teilnahmen (absolute Häufigkeiten, prozentuale Verteilungen in Klammern)

\begin{tabular}{|c|c|c|c|c|}
\hline \multirow[t]{2}{*}{ Partizipationsaspekt } & \multirow{2}{*}{$\begin{array}{l}\text { Gültige } \\
\text { Antworten }\end{array}$} & \multicolumn{2}{|c|}{ Einschätzung } & \multirow[t]{2}{*}{ Summe } \\
\hline & & $\mathrm{Ja}$ & Nein & \\
\hline Einwilligung in die Teilnahme & 176 & $\begin{array}{l}141 \\
(80,1 \%)\end{array}$ & $\begin{array}{l}35 \\
(19,9 \%)\end{array}$ & $\begin{array}{l}176 \\
(100,0 \%)\end{array}$ \\
\hline $\begin{array}{l}\text { Möglichkeit der Mitbestimmung von Rahmen- } \\
\text { bedingungen }\end{array}$ & 162 & $\begin{array}{l}44 \\
(27,2 \%)\end{array}$ & $\begin{array}{l}118 \\
(72,8 \%)\end{array}$ & $\begin{array}{l}162 \\
(100,0 \%)\end{array}$ \\
\hline $\begin{array}{l}\text { Beachtung der Interessen und Themen der } \\
\text { Kinder }\end{array}$ & 155 & $\begin{array}{l}89 \\
(57,4 \%)\end{array}$ & $\begin{array}{l}66 \\
(42,6 \%)\end{array}$ & $\begin{array}{l}155 \\
(100,0 \%)\end{array}$ \\
\hline $\begin{array}{l}\text { Abstimmung der Erhebungsmethode auf } \\
\text { sprachliche und nicht-sprachliche Ausdrucks- } \\
\text { möglichkeiten }\end{array}$ & 175 & $\begin{array}{l}160 \\
(91,4 \%)\end{array}$ & $\begin{array}{l}15 \\
(8,6 \%)\end{array}$ & $\begin{array}{l}175 \\
(100,0 \%)\end{array}$ \\
\hline $\begin{array}{l}\text { Beachtung der Muttersprache (falls erforder- } \\
\text { lich) }\end{array}$ & 138 & $\begin{array}{l}85 \\
(61,6 \%)\end{array}$ & $\begin{array}{l}53 \\
(38,4 \%)\end{array}$ & $\begin{array}{l}138 \\
(100,0 \%)\end{array}$ \\
\hline$\Sigma$ & $\begin{array}{l}630 \\
(100,0 \%)\end{array}$ & $\begin{array}{l}378 \\
(60,0 \%)\end{array}$ & $\begin{array}{l}252 \\
(40,0 \%)\end{array}$ & $\begin{array}{l}630 \\
(100,0 \%)\end{array}$ \\
\hline
\end{tabular}

${ }^{a}$ Im Fragebogen wurde den Teilnehmenden dazu folgende Frage gestellt: Bitte denken Sie an das letzte Forschungsprojekt, an dem Kinder Ihrer Gruppe beteiligt waren. Wurden die Kinder in den folgenden Punkten in die Untersuchung einbezogen?

$\left.S D=1,10, \sigma^{2}=1,23\right)$. Aufgrund der Anzahl der Skalenitems kann der Index einen Wert zwischen 0,0 bis 6,0 annehmen. Analog zur Beschreibung des Grades der ethischen Strenge in Projekten mit Kindern auf der Grundlage von $\mathrm{I}_{\mathrm{FESKi}}$ (siehe oben), kann auch der Index der Partizipation von Kindern $\mathrm{I}_{\text {PartK }}$ zur Beschreibung von Graden der Partizipation von Kindern genutzt werden. Auf der Grundlage des skalenbezogenen Indexes wird die Einteilung von vier Stufen vorgeschlagen: Nicht vorhanden $\left(\mathrm{I}_{\mathrm{PartK}}=0,0\right)$, niedrig $\left(0,5 \leq \mathrm{I}_{\mathrm{PartK}} \geq 2,0\right)$, mittel $\left(2,0<\mathrm{I}_{\mathrm{Part} \mathrm{K}} \geq 4,0\right)$ und hoch $\left(4,0<\mathrm{I}_{\text {PartK }} \geq 6,0\right)$. In Bezug auf diese Einteilung der Grade zeigt sich, dass die von den pädagogischen Fach- und Leitungskräften eingeschätzten Forschungsprojekte im Durchschnitt einen mittleren Grad der Partizipation der Kinder aufweisen.

Von Interesse war, in wieweit ein Zusammenhang zwischen der ethischen Strenge und der Einräumung von Partizipationsmöglichkeiten besteht. Dazu wurden die Zusammenhangsmaße zwischen dem berechneten Index $\mathrm{I}_{\mathrm{FESK}}$ und dem Skalenitem Möglichkeiten der Mitbestimmung von Rahmenbedingungen (Stufe 6 des Partizipationsmodells) ermittelt. Der berechnete Eta-Koeffizient $(0,537)$ legt eine Abhängigkeit der Variablen Möglichkeiten der Mitbestimmung von Rahmenbedingungen von der Höhe des Indexwertes der ethischen Strenge der Forschung mit Kindern $\mathrm{I}_{\mathrm{FESK}}$ nahe $\left(\chi^{2}\right.$ exakt $\left.=43,54 * *, p=0,081, d f=15\right)$, weshalb die Nullhypothese abgelehnt wird. Auf der Grundlage der Daten ist zu schließen, dass die Einräumung der Partizipationsmöglichkeit von Kindern (Mitbestimmung der Rahmenbedingungen) im Forschungsprojekt dann häufiger als zutreffend von den befragten Fach- und Leitungskräften eingeschätzt wurde, wenn der Index-Wert $\mathrm{I}_{\mathrm{FESK}}$ einen höheren Wert erreichte. 


\section{Interpretation der Ergebnisse}

Die Ergebnisse der Kinderinterviews zeigen bezugnehmend auf die Partizipation im Forschungsprozess eine erlebte Forschungspraxis, die Artikel 12 und Artikel 13 der UN-Kinderrechtskonvention zum Teil entspricht: Die Kinder wurden aus ihrer Sicht teils informiert, angehört und einbezogen, teilweise aber auch keine Einflussnahme auf den (strukturellen) Forschungsprozess zugestanden. Das zeigt sich insbesondere für die Freiwilligkeit der Teilnahme und die informierte Einwilligung. Die Erfahrungen der Kinder variieren dahingehend zwischen einer Nicht-Partizipation (als erlebte Anweisung) bis zur Partizipation (Einbezug in den Entscheidungsprozess durch Gatekeeper). Somit erscheinen für eine „erinnerungswürdige“ Forschungserfahrung für Kinder ihre aktive Rolle und Einbeziehung sowie Mitbestimmung im Forschungsprozess voraussetzungsvoll zu sein.

Die Ergebnisse der Online-Befragung untermauern dabei die Schilderungen der Kinder. Aus der Sicht der befragten Fach- und Leitungskräfte werden die Rechte der Kinder durch Forscher/-innen nur zu einem mittleren Grad gewahrt: Sowohl der Index-Wert der ethischen Strenge $\mathrm{I}_{\mathrm{FESKi}}$ als auch der Index-Wert der Partizipation $\mathrm{I}_{\text {PartK }}$ weisen in der Erhebung mittlere Werte auf. Beginnend mit der direkten Einholung des informierten Einverständnisses bis hin zur Realisierung einer tatsächlichen Partizipation der Kinder spiegeln die Einschätzungen der pädagogischen Fach- und Leitungskräfte nur ein mittleres Niveau wider. Die Erreichung einer ethischen Symmetrie zwischen Forscher/-innen und Kindern sowie die Realisierung einer echten Partizipation (ab Stufe 6 des Partizipationsmodells) lässt sich für die von den Befragten eingeschätzten Forschungsprojekte in Kitas anhand der Daten nicht feststellen. Vielmehr weisen die Ergebnisse darauf hin, dass die Kinderrechte in Forschungsprojekten aus der Sicht der befragten Personen nicht flächendeckend und nicht hinsichtlich aller Kriterien, die sich aus dem Ethik-Kodex (DGfE 2016) und der UN-Kinderrechtskonvention ergeben (Artikel 12, Artikel 13), gewahrt werden. Diese Erkenntnisse decken sich mit den Ergebnissen internationaler Studien (Jacquez et al. 2013; Raffety 2015; von Unger 2014), die eine vorwiegend geringe Partizipation von Kindern in Forschungsprojekten aufzeigen und die Kinderrechte als nicht hinreichend gewahrt konstatieren (Canosa et al. 2018; Christensen und Prout 2002).

Bezugnehmend auf den Titel des Beitrags ist auf der Grundlage der vorgestellten Ergebnisse zu schlussfolgern, dass Kindern im Rahmen von Forschungsprojekten in Kitas eher eine Minimalbeteiligung eingeräumt wird.

Vertiefende Studien sollten durchgeführt werden, um gezielter die Wahrung der Kinderrechte und der Partizipation der Kinder auch aus der Perspektive der Forscher/-innen zu erfassen. Zudem sollten die Ursachen einer Nicht-Wahrung der Kinderrechte eruiert werden, um Handlungsmöglichkeiten für die stringentere Sicherung der Rechte von Kindern erarbeiten zu können. 


\section{Limitationen}

Mit Blick auf die Güte der Studien sind einige Limitationen anzuführen, die sich auf das methodische Vorgehen beziehen. So wurde bei der Online-Befragung der Zugang und Umgang mit neuen Medien aufseiten der Teilnehmer/-innen vorausgesetzt. Zugleich erfolgte keine Einflussnahme auf den Zugang der pädagogischen Fachkräfte, d.h. es ist unklar, inwieweit die Kita-Leitungen die Information zum Forschungsprojekt an ihre Mitarbeiter/-innen weiterleiteten. Zudem ist festzuhalten, dass von den 1200 Teilnehmer/-innen nur $n=670(55,8 \%)$ die Befragung vollständig bearbeitet haben. Hinsichtlich der Kinderstudie ist anzumerken, dass vereinzelt keine kindgerechten Nachfragen der Interviewer/-innen zu forschungsrelevanten Äußerungen der Kinder erfolgten.

Funding Open Access funding enabled and organized by Projekt DEAL.

Open Access Dieser Artikel wird unter der Creative Commons Namensnennung 4.0 International Lizenz veröffentlicht, welche die Nutzung, Vervielfältigung, Bearbeitung, Verbreitung und Wiedergabe in jeglichem Medium und Format erlaubt, sofern Sie den/die ursprünglichen Autor(en) und die Quelle ordnungsgemäß nennen, einen Link zur Creative Commons Lizenz beifügen und angeben, ob Änderungen vorgenommen wurden.

Die in diesem Artikel enthaltenen Bilder und sonstiges Drittmaterial unterliegen ebenfalls der genannten Creative Commons Lizenz, sofern sich aus der Abbildungslegende nichts anderes ergibt. Sofern das betreffende Material nicht unter der genannten Creative Commons Lizenz steht und die betreffende Handlung nicht nach gesetzlichen Vorschriften erlaubt ist, ist für die oben aufgeführten Weiterverwendungen des Materials die Einwilligung des jeweiligen Rechteinhabers einzuholen.

Weitere Details zur Lizenz entnehmen Sie bitte der Lizenzinformation auf http://creativecommons.org/ licenses/by/4.0/deed.de.

\section{Literatur}

Arbeitsgemeinschaft für Kinder- und Jugendhilfe (AGJ) (2020). Partizipative Forschung - ein Forschungskonzept für die Kinder- und Jugendhilfe? Positionspapier der Arbeitsgemeinschaft für Kinder- und Jugendhilfe - AGJ. https://www.agj.de/fileadmin/files/positionen/2020/Partizipative_Forschung.pdf. Zugegriffen: 29. Okt. 2020.

Ballaschk, I., \& Anders, A. (2020). Partizipation aus der Perspektive von Kindern. Frühe Bildung, 9(1), 3-8.

Bruner, C.F., Winklhofer, U., \& Zinser, C. (2001). Partizipation - Ein Kinderspiel? Beteiligungsmodelle in Kindertagesstätten, Schulen, Kommunen und Verbänden. Berlin: DJI. Herausgegeben vom Bundesministerium für Familie, Senioren, Frauen und Jugend

Canosa, A., Graham, A., \& Wilson, E. (2018). Reflexivity and ethical mindfulness in participatory research with children: what does it really look like? Childhood, 25(3), 400-415.

Cheney, K.E. (2011). Children as ethnographers: Reflections on the importance of participatory research in assessing orphans' needs. Childhood, 18(2), 166-179.

Christensen, P., \& Prout, A. (2002). Working with ethical symmetry in social research with children. Childhood, 9(4), 477-497.

Deutsche Gesellschaft für Erziehungswissenschaft (DGfE) (2016). Ethik-Kodex der Deutschen Gesellschaft für Erziehungswissenschaft (DGfE). https://www.dgfe.de/fileadmin/OrdnerRedakteure/ Satzung_etc/Ethikkodex_2016.pdf. Zugegriffen: 29. Okt. 2020.

Döring, N. (2015). Qualitätskriterien für quantitative empirische Studien. In S. Maschke \& L. Stecher (Hrsg.), Methoden der empirischen erziehungswissenschaftlichen Forschung, Qualitätskriterien in 
der empirischen Forschung. Enzyklopädie Erziehungswissenschaft Online. (S. 1-39). https://doi.org/ 10.3262/EEO07150345.

Döring, N., \& Bortz, J. (2016a). Qualitätskriterien in der empirischen Sozialforschung. In N. Döring \& J. Bortz (Hrsg.), Forschungsmethoden und Evaluation in den Sozial- und Humanwissenschaften (5. Aufl. S. 81-119). Berlin: Springer.

Döring, N., \& Bortz, J. (2016b). Forschungs- und Wissenschaftsethik. In N. Döring \& J. Bortz (Hrsg.), Forschungsmethoden und Evaluation in den Sozial- und Humanwissenschaften (5. Aufl. S. 122-139). Berlin: Springer.

Heinzel, F. (2012). Einleitung. In F. Heinzel (Hrsg.), Methoden der Kindheitsforschung. Ein Überblick über Forschungszugänge zur kindlichen Perspektive (2. Aufl. S. 9-12). Weinheim, Basel: Beltz Juventa.

Horgan, D. (2017). Child participatory research methods: Attempts to go 'deeper. Childhood, 24(2), 245-259.

Jacquez, F., Vaughn, L. M., \& Wagner, E. (2013). Youth as partners, participants or passive recipients: a review of children and adolescents in community-based participatory research (CBPR). American Journal of Community Psychology, 51, 176-183.

Kelle, U. (2014). Mixed Methods. In N. Baur \& J. Blasius (Hrsg.), Handbuch Methoden der empirischen Sozialforschung (S. 154-166). Wiesbaden: Springer VS.

Kuckartz, U. (2018). Qualitative Inhaltsanalyse. Methoden, Praxis, Computerunterstützung (4. Aufl.). Weinheim, Basel: Beltz Juventa.

Leiner, D. J. (2019). SoSci Survey (Version 3.1.06) [Computer software]. https://www.soscisurvey.de. Zugegriffen: 29. Okt. 2020.

Moser, W. (2012). Kinderrechte: Empowerment durch Forschung. Partizipatorische Forschung im Kontext der Kinderrechtskonvention. beziehungsweise, 11, 1-4.

Naidoo, J., \& Wills, J. (2003). Lehrbuch der Gesundheitsförderung. Umfassend und anschaulich mit vielen Beispielen und Projekten aus der Praxis der Gesundheitsförderung. Köln: BZgA.

Nentwig-Gesemann, I., \& Großmaß, R. (2017). Kinder als Forschungssubjekte - von den rechtlichen und forschungsethischen Grundlagen zur forschungspraktischen Realisierung. In I. Nentwig-Gesemann \& K. Fröhlich-Gildhoff (Hrsg.), Forschung in der Frühpädagogik X. Zehn Jahre frühpädagogische Forschung - Bilanzierungen und Reflexionen (S. 209-227). Freiburg i. Br.: FEL Verlag Forschung Entwicklung - Lehre.

Phelan, S. K., \& Kinsella, E. A. (2013). Picture this ... safety, dignity, and voice-ethical research with children: practical considerations for the reflexive researcher. Qualitative Inquiry, 19(2), 81-90.

Porst, R. (2014). Fragebogen. Ein Arbeitsbuch (4. Aufl.). Wiesbaden: Springer VS.

Pospeschill, M. (2013). Empirische Methoden in der Psychologie. München: Ernst Reinhardt.

Raffety, E. L. (2015). Minimizimg social distance: participatory research with children. Childhood, 22(3), 409-422.

Rat für Sozial- und Wirtschaftsdaten (RatSWD) (2017). Forschungsethische Grundsätze und Prüfverfahren in den Sozial- und Wirtschaftswissenschaften. RatSWD. https://doi.org/10.17620/02671.1.

Statistisches Bundesamt (Destatis) (2020). Statistiken der Kinder- und Jugendhilfe. Kinder und tätige Personen in Tageseinrichtungen und in öffentlich geförderter Kindertagespflege am 1.3.2020. https://www.destatis.de/DE/Themen/Gesellschaft-Umwelt/Soziales/Kindertagesbetreuung/_inhalt. html;jsessionid=87A09F70DC8E4CCC83507701AB0C9DF1.internet8732\#sprg234640. Zugegriffen: 29. Okt. 2020.

Thomas, N., \& O'Kane, C. (1998). The ethics of participatory research with children. Children \& Society, $12,336-348$.

Trautmann, T. (2010). Interviews mit Kindern. Grundlagen, Techniken, Besonderheiten, Beispiele. Wiesbaden: VS.

von Unger, H. (2014). Partizipative Forschung. Einführung in die Forschungspraxis. Wiesbaden: Springer.

Vogl, S. (2015). Interviews mit Kindern führen. Eine praxisorientierte Einführung. Weinheim, Basel: Beltz Juventa.

Wilkinson, C., \& Wilkinson, S. (2017). Doing it write: representation and responsibility in writing up participatory research involving young people. Social Inclusion, 5(3), 219-227.

Wright, M. T., von Unger, H., \& Block, M. (2010). Partizipation der Zielgruppe in der Gesundheitsförderung und Prävention. In M. T. Wright (Hrsg.), Partizipative Qualitätsentwicklung in der Gesundheitsförderung und Prävention (S. 35-52). Bern: Huber. 